\title{
Experience of Holding Epilepsy Detection and Management Multidisciplinary Camp Organized in Alibaug, Maharashtra during the COVID 19 Pandemic: A Joint Project by Epilepsy Foundation and National Health Mission, Government of Maharashtra
}

\section{Nirmal Surya ${ }^{1 *}$, Hitav Someshwar², BN Sawant ${ }^{3}$, Santosh Kondekar, Saurabh Kamath ${ }^{3}$, Mehul Desai ${ }^{3}$, Arti Sharma ${ }^{5}$, Janvi Someshwar ${ }^{6}$ and Pooja Nagpure ${ }^{7}$}

${ }^{1}$ Founder Trustee and Chairman, Epilepsy Foundation India, Mumbai, India

${ }^{2}$ Physiotherapist, Epilepsy Foundation India, Mumbai, India

${ }^{3}$ Neurologist, Epilepsy Foundation India, Mumbai, India

${ }^{4}$ Pediatrician, Epilepsy Foundation India, Mumbai, India

${ }^{5}$ Clinical Psychologist, Epilepsy Foundation India, Mumbai, India

${ }^{6}$ Occupational Therapist, Epilepsy Foundation India, Mumbai, India

${ }^{7}$ Speech Therapist, Epilepsy Foundation India, Mumbai, India

*Corresponding Author: Nirmal Surya, Founder Trustee and Chairman, Epilepsy

Foundation India, Mumbai, India.
Received: March 30, 2021

Published: April 16, 2021

(C) All rights are reserved by Nirmal Surya., et al.
There are approximately 13 million people suffering from Epilepsy in India and the treatment gap is 50 - 75\% largely due to myths and taboo associated with Epilepsy [1]. About 70\% patients are treatable with single medicine if the treatment is started in time. Epilepsy Foundation India, established in the year 2009, is a 'Non Profit' organization and registered under sections $12 \mathrm{~A}$ and $80 \mathrm{G}$ of the Income Tax Act, 1961. It is dedicated to 'Cure and Rehabilitation of Patients suffering from Epilepsy', spreading awareness and educating the people about Epilepsy [2].

Epilepsy Foundation India in Association with National Health Mission (NHM) as a public private partner Has developed "the model of total epilepsy care for rural districts of Maharashtra" through this they conduct regular Monthly Camp in Maharashtra for detection and treatment of epilepsy. Since 2011 we have organised 88 camps which were very successful and we saw a total of 31,655 patients suffering from epilepsy. During the camps, a team of 10 neurologist and neurosurgeons along with two physiotherapists, occupational therapists, speech and language pathologist and a psychologist. The team also consists of EEG technicians, People with epilepsy and relatives, Social workers and street play artists. During the camps, the main aim is to provide comprehensive epilepsy care and also spread awareness about the condition and curb the myths and misbelieves of the society on epilepsy. We aim to reduce the social stigma associated with Epilepsy and thereby increasing the quality life of the persons with epilepsy. Patient and caregiver awareness about the epilepsy are also assessed during the camps $[2,3]$.

The $89^{\text {th }}$ Camp was scheduled on $15^{\text {th }}$ March 2020 at Buldana was postponed due to the COVID 19 Pandemic. COVID 19 Pandemic has caused grave financial, economical and psychological stress to normal as well as diseased population. Due to the pandemic the camps were stopped for a period of 1 year. During this duration the patient's seizure had increased due to an increase in anxiety and stress, non follow up and non availability of medications. Epilepsy foundation provided with free medications for people with epilepsy in the rural districts of Maharashtra during the pandemic. The foundation also provided free teleconsultation and management by all the members of the multidisciplinary group [4]. These services were availed by many, but a majority of the patients were unable to connect online belonged to the rural districts of Maharashtra who 
either did not have smart phones, had limited knowledge on the use of technology or/and Low bandwidth of internet connectivity [4].

Due to the problems faced by the patients in the rural districts of Maharashtra, Epilepsy foundation India held a joint meeting with the National Health Mission, Government of Maharashtra to organize a medical camp for detection and treatment of epilepsy amid the COVID 19 pandemic. On 21st March 2021, the 89 ${ }^{\text {th }}$ rural camp was held at civil hospital, Alibaug with the coordination among Epilepsy foundation team, National health mission Government of Maharashtra and the local governing body of Alibaug. This was the first Epilepsy camp held at a civil hospital during the covid 19 pandemic in Maharashtra. A team of 4 neurologists, 1 pediatrician, 3 EEG technicians, 1 Physiotherapist, 1 occupational therapist, 1 speech therapist and 1 clinical psychologist along with 3 social workers were present for a one day medical camp. We followed all measures of prevention as prescribed by the ministry of health and family welfare. All the members of the team were thermal scanning and tested for rapid antigen upon arrival, after testing nega- tive the team members were given individual cabins with a fresh set of masks and personal protective equipments with the nursing staff stationed at each cabin maintaining social distancing among patients visiting different members.

On arrival to the civil hospital the patients were first thermal scanned and then later registered at the registration desk on the ground floor followed by which they were sent to the $1^{\text {st }}$ floor for consultation with Neurologist in 4 different cabins, the patients were examined by the neurologists keeping in mind the COVID 19 prevention protocols. The Patients Requiring EEG were sent to the EEG room and those who required OT, PT, ST or Counseling were sent to the ground floor. After finishing the required therapies, the patients were sent to the medication and documentation room (Figure 1). All the patients documents were scanned; they were provided with three months of free anti epileptic medications. Those patients requiring EEG and CT scan were provided the same at free of cost during the camp. A generous donor of Epilepsy foundation provided free clothes to young girls as well as the elder females.

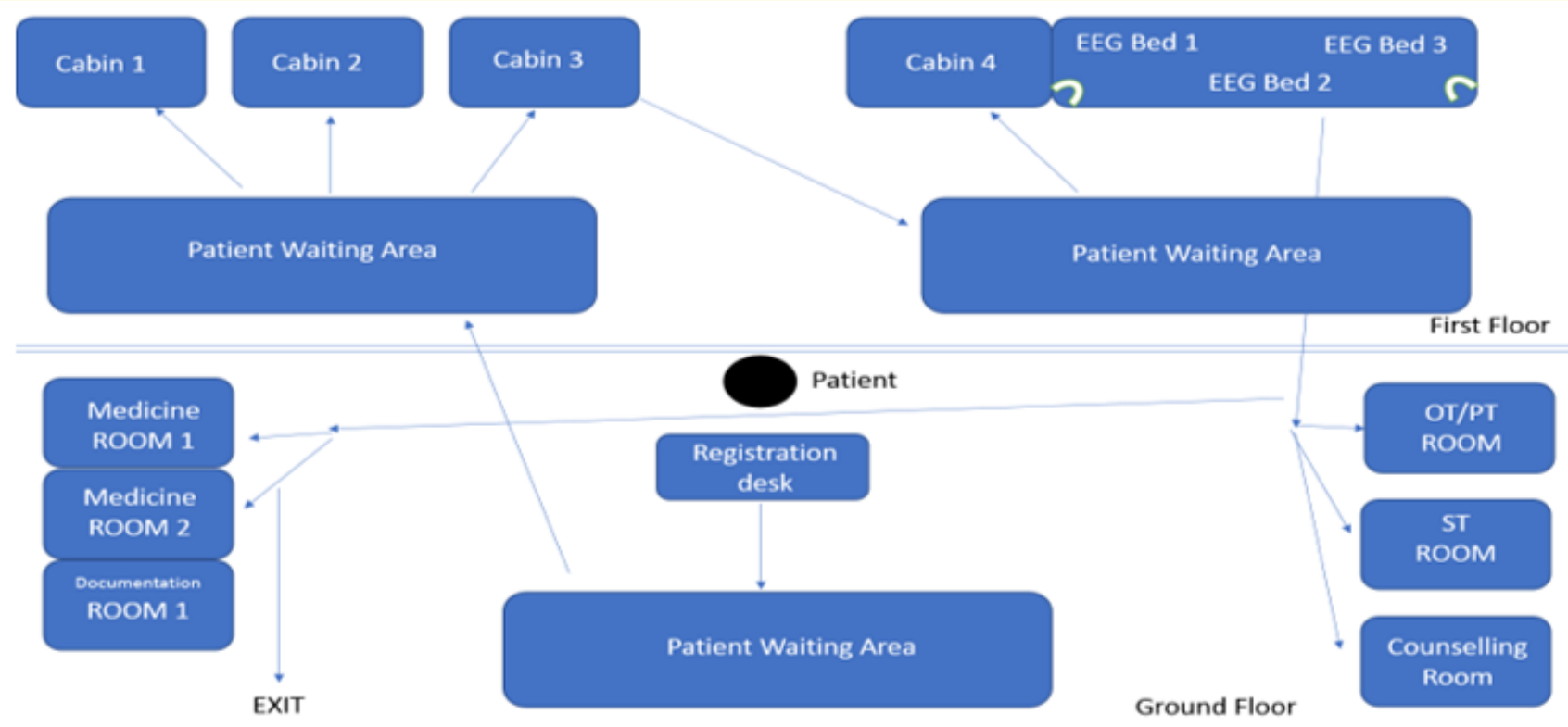

Figure 1: Floor plan during the camp. 
As per the model, Epilepsy foundation organizes one day CME lectures and hands on training on epilepsy during the camp. These CMEs are accredited by Maharashtra Medical Council for two points to provide update on epilepsy care to physicians and medical officers. The permission to conduct the CME was obtained for 50 participants from the district collector while maintaining proper physical distancing.

All measures of social distancing and prevention taken keeping in mind the guidelines of COVID 19 prevention. A total of 89 patients suffering from epilepsy were examined by the team of doctors and Therapist. An EEG room was set up with 3 EEG Machines by 4 EEG technicians. A total of 25 EEGs were done. Among the patients were people who had come from various districts and Schools Health workers as well as local people from Alibaug district. Besides epilepsy patients there were few patients suffering from MR with epilepsy and other disabilities. A Team consisting of a Clinical Psychologist, Physiotherapist, Occupational Therapist and Speech Therapists from Mumbai were also present to evaluate and treat patients. They treated 44 Patients overall. ( $\mathrm{CP}=19$, $\mathrm{PT}=08, \mathrm{OT}=10$ and $\mathrm{ST}=07$. Every Patient's data was recorded for future reference and follow up. All the patients were given free anti epileptic drugs for three months and five patients underwent free CT scan of the brain. Due to COVID 19 restrictions, street play to create awareness about epilepsy in different locations of the city was not held during this camp.

\section{Conclusion}

Conducting Epilepsy and other diseases camps is feasible and safe even during the covid 19 pandemic, provided proper precautions such as wearing masks and other protective equipment, Hand hygiene and social distancing is followed during such camps. In all the camp was a success, the smiles on the persons with epilepsy post consultation, free medication, EEGs and CT scan brought great satisfaction to the Epilepsy Foundation India team.

\section{Acknowledgment}

We would like to thank National health mission, Government of Maharashtra, Civil Surgeon at the civil hospital in Alibaug, Medical and nursing staff of the civil hospital along with the EEG technicians and Social Workers of Epilepsy Foundation India for their constant support to make this camp a safe and successful camp.

\section{Bibliography}

1. Santhosh NS., et al. "Epilepsy: Indian perspective. Annals of Indian Academy of Neurology 17 (2014): S3-S11.

2. Nirmal Surya., et al. "Quality of Life assessment of people with Epilepsy in COVID Pandemic". International Journal of Psychiatry 5.2 (2020).

3. Surya N and Someshwar H. "Community awareness and attitudes towards Epilepsy in rural districts of Maharashtra, India: A questionnaire survey". EC Neurology 11.8 (2019): 592600.

4. Nirmal Surya., et al. "Comprehensive epilepsy care during the lockdown due to covid india: "epilepsy foundation india model". International Journal of Current Research 12.7 (2020): 12159-12162.

\section{Assets from publication with us}

- Prompt Acknowledgement after receiving the article

- Thorough Double blinded peer review

- Rapid Publication

- Issue of Publication Certificate

- High visibility of your Published work

Website: www.actascientific.com/

Submit Article: www.actascientific.com/submission.php Email us: editor@actascientific.com

Contact us: +919182824667 